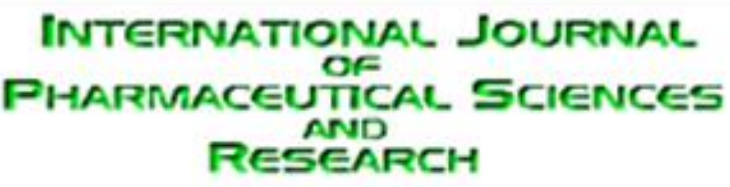

Received on 04 January, 2013; received in revised form, 23 February, 2013; accepted, 21 April, 2013

\title{
POLIO: A REVIEW
}

Robin Singh*, Amit K. Monga and Souravh Bais

Rayat Institute of Pharmacy, Railmajra, Distt. S.B.S. Nagar- 144533, Punjab, India

Keywords:

Polio, Vaccine, Herbal medicine, Immunity

\section{Correspondence to Author:}

\section{Robin Singh}

Rayat Institute of Pharmacy, Railmajra, Distt. S.B.S. Nagar- 144533, Punjab, India

E-mail:souravh2008.123@redifamil.com

\begin{tabular}{|l|c|}
\hline QUICK RESPONSE CODE & $\begin{array}{c}\text { IJPSR: } \\
\text { ICV (2011)- 5.07 }\end{array}$ \\
\cline { 4 - 4 } & $\begin{array}{c}\text { Article can be } \\
\text { accessed online on: } \\
\text { www.ijpsr.com }\end{array}$ \\
\hline
\end{tabular}

ABSTRACT: Postpolio syndrome (PPS) may affect survivors of paralytic poliomyelitis and is characterised by a complex of neuromuscular symptoms leading to a decline in physical functioning. The effectiveness of pharmacological treatment and rehabilitation management in PPS is not yet established. To review systematically the effects of any treatment for PPS compared to placebo, usual care or no treatment. Postpolio syndrome is characterized by the exacerbation of existing or new health problems, most often muscle weakness and fatigability, general fatigue, and pain, after a period of stability subsequent to acute polio infection. Diagnosis is based on the presence of a lower motor neuron disorder that is supported by neurophysiological findings, with exclusion of other disorders as causes of the new symptoms. The muscle-related effects of postpolio syndrome are possibly associated with an ongoing process of denervation and reinnervation, reaching a point at which denervation is no longer compensated for by reinnervation. The cause of this denervation is unknown, but an inflammatory process is possible. Rehabilitation in patients with postpolio syndrome should take a multi professional and multidisciplinary approach, with an emphasis on physiotherapy, including enhanced or individually modified physical activity, and muscle training. Patients with postpolio syndrome should be advised to avoid both inactivity and over use of weak muscles. Evaluation of the need for orthoses and assistive devices is often required.

\section{INTRODUCTION: Poliomyelitis is an} acute, viral, infectious disease spread from person to person, primarily via the fecal-oral route. Polio is a contagious disease caused by an intestinal virus that may attack nerve cells of the brain and spinal cord. Symptoms include fever, headache, sore throat, and vomiting. Some victims develop neurological complications, including stiffness of the neck and back, weak muscles, pain in the joints, and paralysis of one or more limbs or respiratory muscles. In severe cases it may be fatal, due to respiratory paralysis. Polio can be spread through contact with contaminated faeces (for example, by changing an infected baby's diapers) or through airborne droplets, in food, or in water.
The virus enters the body by nose or mouth, and then travels to the intestines where it incubates. Next, it enters the bloodstream where anti-polio antibodies are produced. In most cases, this stops the progression of the virus and the individual gains permanent immunity against the disease ${ }^{1}$.

Although approximately $90 \%$ of polio infections cause no symptoms at all, affected individuals can exhibit a range of symptoms if the virus enters the blood stream. In about $1 \%$ of cases, the virus enters the central nervous system, preferentially infecting and destroying motor neurons, leading to muscle weakness and acute flaccid paralysis. 
Different types of paralysis may occur, depending on the nerves involved. Spinal polio is the most common form, characterized by asymmetric paralysis that most often involves the legs. Bulbar polio leads to weakness of muscles innervated by cranial nerves. Bulbospinal polio is a combination of bulbar and spinal paralysis ${ }^{2}$.

Poliomyelitis was first recognized as a distinct condition by Jakob Heine in 1840. Its causative agent, poliovirus, was identified in 1908 by Karl Landsteiner. Although major polio epidemics were unknown before the late 19th century, polio was one of the most dreaded childhood diseases of the 20th century. Polio epidemics have crippled thousands of people, mostly young children; the disease has caused paralysis and death for much of human history. Polio had existed for thousands of years quietly as an endemic pathogen until the 1880s, when major epidemics began to occur in Europe; soon after, widespread epidemics appeared in the United States ${ }^{3}$.

By 1910, much of the world experienced a dramatic increase in polio cases and epidemics became regular events, primarily in cities during the summer months. These epidemics-which left thousands of children and adults paralyzed-provided the impetus for a "Great Race" towards the development of a vaccine. Developed in the 1950s, polio vaccines are credited with reducing the global number of polio cases per year from many hundreds of thousands to today under a thousand. Enhanced vaccination efforts led by Rotary International, the World Health Organization, and UNICEF should result in global eradication of the disease ${ }^{4}$.

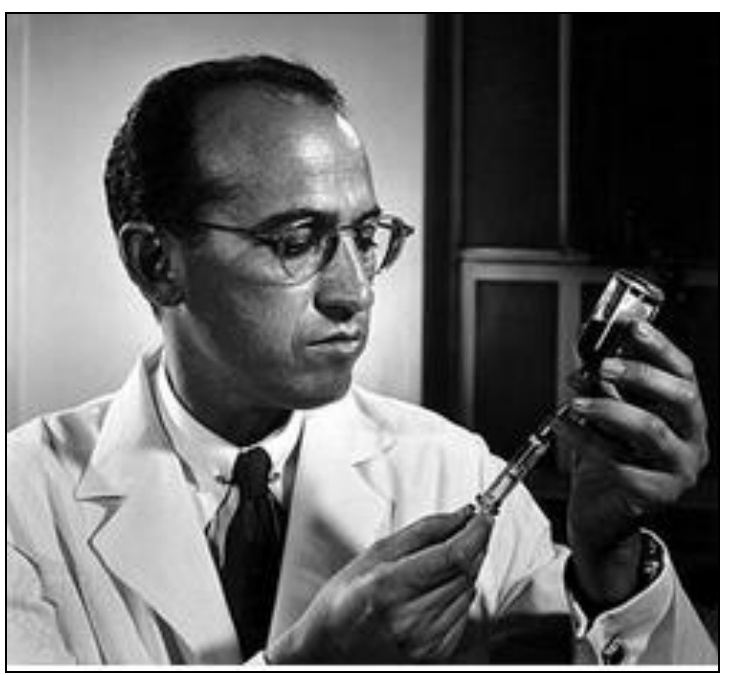

FIG. 1: Dr. JONAS SALK- INVENTOR OF POLIO VACCINE
CAUSE: Poliomyelitis is caused by infection with a member of the genus Enterovirus known as poliovirus (PV). This group of RNA viruses colonize the gastrointestinal tract- specifically the oropharynx and the intestine. The incubation time (to the first signs and symptoms) ranges from three to 35 days, with a more common span of six to 20 days PV infects and causes disease in humans alone. Its structure is very simple, composed of a single (+) sense RNA genome enclosed in a protein shell called a capsid.

In addition to protecting the virus's genetic material, the capsid proteins enable poliovirus to infect certain types of cells. Three serotypes of poliovirus have been identified-poliovirus type 1 (PV1), type 2 (PV2), and type 3 (PV3)-each with a slightly different capsid protein. All three are extremely virulent and produce the same disease symptoms. PV1 is the most commonly encountered form, and the one most closely associated with paralysis ${ }^{5}$.

Individuals who are exposed to the virus, either through infection or by immunization with polio vaccine, develop immunity. In immune individuals, IgA antibodies against poliovirus are present in the tonsils and gastrointestinal tract, and are able to block virus replication; IgG and IgM antibodies against PV can prevent the spread of the virus to motor neurons of the central nervous system. Infection or vaccination with one serotype of poliovirus does not provide immunity against the other serotypes, and full immunity requires exposure to each serotype ${ }^{6}$.

\section{TYPE OF POLIO:}

Paralytic polio: In around $1 \%$ of infections, poliovirus spreads along certain nerve fiber pathways, preferentially replicating in and destroying motor neurons within the spinal cord, brain stem, or motor cortex. This leads to the development of paralytic poliomyelitis, the various forms of which (spinal, bulbar, and bulbospinal) vary only with the amount of neuronal damage and inflammation that occurs, and the region of the CNS affected. The destruction of neuronal cells produces lesions within the spinal ganglia; these may also occur in the reticular formation, vestibular nuclei, cerebellar vermis, and deep cerebellar nuclei. Inflammation associated with nerve cell destruction often alters the colour and appearance of the gray matter in the 
spinal column; causing it to appear reddish and swollen other destructive changes associated with paralytic disease occur in the forebrain region, specifically the hypothalamus and thalamus. The molecular mechanisms by which poliovirus causes paralytic disease are poorly understood ${ }^{7}$.

Early symptoms of paralytic polio include high fever, headache, stiffness in the back and neck, asymmetrical weakness of various muscles, sensitivity to touch, difficulty swallowing, muscle pain, loss of superficial and deep reflexes, paresthesia (pins and needles), irritability, constipation, or difficulty urinating. Paralysis generally develops one to ten days after early symptoms begin, progresses for two to three days, and is usually complete by the time the fever breaks 8

The likelihood of developing paralytic polio increases with age, as does the extent of paralysis. In children, non-paralytic meningitis is the most likely consequence of CNS involvement, and paralysis occurs in only one in 1000 cases. In adults, paralysis occurs in one in 75 cases ${ }^{9}$. In children under five years of age, paralysis of one leg is most common; in adults, extensive paralysis of the chest and abdomen also affecting all four limbs-quadriplegia-is more likely. Paralysis rates also vary depending on the serotype of the infecting poliovirus; the highest rates of paralysis (one in 200) are associated with poliovirus type 1 , the lowest rates (one in 2,000) are associated with type $2^{10}$.

Spinal polio: Spinal polio, the most common form of paralytic poliomyelitis, results from viral invasion of the motor neurons of the anterior horn cells, or the ventral (front) gray matter section in the spinal columns, which are responsible for movement of the muscles, including those of the trunk, limbs and the intercostals muscles.

Virus invasion causes inflammation of the nerve cells, leading to damage or destruction of motor neuron ganglia. When spinal neurons die, wallerian degeneration takes place, leading to weakness of those muscles formerly innervated by the now-dead neurons. With the destruction of nerve cells, the muscles no longer receive signals from the brain or spinal cord; without nerve stimulation, the muscles atrophy, becoming weak, floppy and poorly controlled, and finally completely paralyzed.
Progression to maximum paralysis is rapid (two to four days), and is usually associated with fever and muscle pain. Deep tendon reflexes are also affected, and are usually absent or diminished; sensation (the ability to feel) in the paralyzed limbs, however, is not affected $^{11}$.

The extent of spinal paralysis depends on the region of the cord affected, which may be cervical, thoracic, or lumbar. The virus may affect muscles on both sides of the body, but more often the paralysis is asymmetrical. Any limb or combination of limbs may be affected-one leg, one arm, or both legs and both arms. Paralysis is often more severe proximally (where the limb joins the body) than distally (the fingertips and toes) ${ }^{12}$.

Bulbar polio: Making up about $2 \%$ of cases of paralytic polio, bulbar polio occurs when poliovirus invades and destroys nerves within the bulbar region of the brain stem. The bulbar region is a white matter pathway that connects the cerebral cortex to the brain stem. The destruction of these nerves weakens the muscles supplied by the cranial nerves, producing symptoms of encephalitis, and causes difficulty breathing, speaking and swallowing. Critical nerves affected are the glossopharyngeal nerve, which partially controls swallowing and functions in the throat, tongue movement and taste; the vagus nerve, which sends signals to the heart, intestines, and lungs; and the accessory nerve, which controls upper neck movement.

Due to the effect on swallowing, secretions of mucus may build up in the airway, causing suffocation. Other signs and symptoms include facial weakness, caused by destruction of the trigeminal nerve and facial nerve, which innervate the cheeks, tear ducts, gums, and muscles of the face, among other structures; double vision; difficulty in chewing; and abnormal respiratory rate, depth, and rhythm, which may lead to respiratory arrest. Pulmonary edema and shockare also possible, and may be fatal ${ }^{13}$.

Bulbospinal polio: Approximately $19 \%$ of all paralytic polio cases have both bulbar and spinal symptoms; this subtype is called respiratory or bulbospinal polio. Here, the virus affects the upper part of the cervical spinal cord (cervical vertebrae C3 through C5), and paralysis of the diaphragm occurs. The critical nerves affected are the phrenic nerve, which drives the diaphragm to inflate the lungs, and 
those that drive the muscles needed for swallowing. By destroying these nerves, this form of polio affects breathing, making it difficult or impossible for the patient to breathe without the support of a ventilator. It can lead to paralysis of the arms and legs and may also affect swallowing and heart functions ${ }^{14}$.

According to WHO, on February 2012 India was taken off from the list of polio endemic countries. As the end of 2012, Polio remains endemic in only three countries: Nigeria, Pakistan and Afghanistan, although it continues to cause in other nearby countries due to restablized transmission.

Pathophysiology: Poliovirus enters the body through the mouth, infecting the first cells with which it comes in contact - the pharynx and intestinal mucosa. It gains entry by binding to an immunoglobulin-like receptor, known as the poliovirus receptor or CD155, on the cell membrane. The virus then hijacks the host cell's own machinery, and begins to replicate. Poliovirus divides within gastrointestinal cells for about a week, from where it spreads to the tonsils (specifically the follicular dendritic cells residing within the tonsilar germinal centers), the intestinal lymphoid tissue including the M cells of Peyer's patches, and the deep cervical and mesenteric lymph nodes, where it multiplies abundantly.
The virus is subsequently absorbed into the bloodstream ${ }^{15}$. Known as viremia, the presence of virus in the bloodstream enables it to be widely distributed throughout the body. Poliovirus can survive and multiply within the blood and lymphatics for long periods of time, sometimes as long as 17 weeks. In a small percentage of cases, it can spread and replicate in other sites, such as brown fat, the reticuloendothelial tissues, and muscle.

This sustained replication causes a major viremia, and leads to the development of minor influenza-like symptoms. Rarely, this may progress and the virus may invade the central nervous system, provoking a local inflammatory response. In most cases, this causes a self-limiting inflammation of the meninges, the layers of tissue surrounding the brain, which is known as non-paralytic aseptic meningitis.

Penetration of the CNS provides no known benefit to the virus, and is quite possibly an incidental deviation of a normal gastrointestinal infection. The mechanisms by which poliovirus spreads to the CNS are poorly understood, but it appears to be primarily a chance event-largely independent of the age, gender, or socioeconomic position of the individual 16

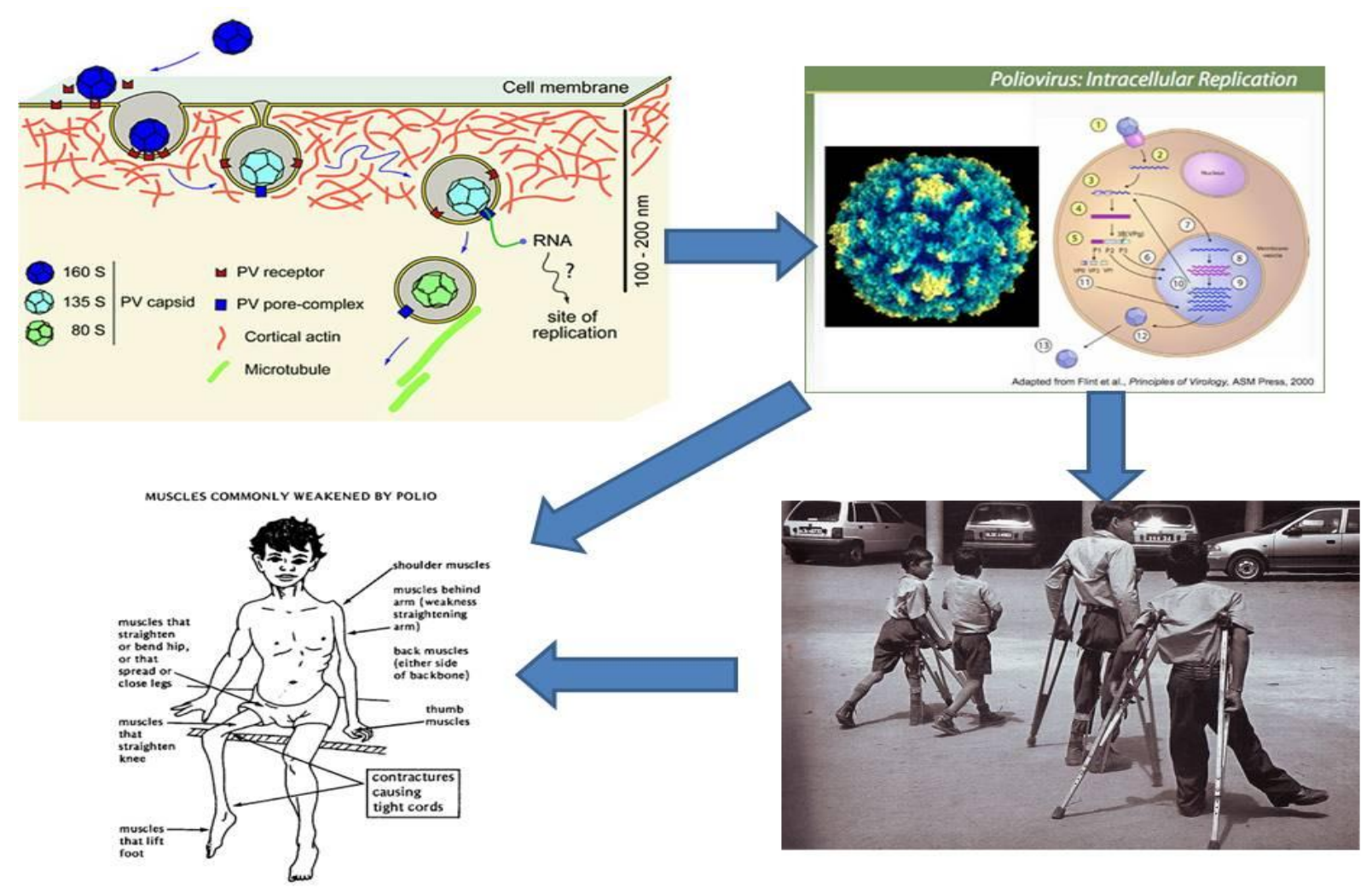

FIG. 3: POLIO VIRUS AND ITS TRANSMISSION 
POLIO VACCINE: Polio vaccines are used throughout the world to combat poliomyelitis (or polio). The first was developed by Jonas Salk and first tested in 1952. Announced to the world by Salk on April 12, 1955, it consists of an injected dose of inactivated (dead) poliovirus. An oral vaccine was developed by Albert Sabin using attenuated poliovirus. Human trials of Sabin's vaccine began in 1957 and it was licensed in $1962^{17}$. Because there is no long term carrier state for poliovirus in immune competent individuals, polioviruses have no nonprimate reservoir in nature, and survival of the virus in the environment for an extended period of time appears to be remote.

Therefore, interruption of person to person transmission of the virus by vaccination is the critical step in global polio eradication. The two vaccines have eliminated polio from most countries in the world and reduced the worldwide incidence from an estimated 350,000 cases in 1988 to 1,652 cases in $2007^{18}$.

Storage condition: Polio virus store in $-200 \mathrm{C}$ for 24 months, 00C for 6 months and 40C for 3 months ${ }^{19}$.

\section{TYPE OF VACCINE:}

Inactivated vaccine: The first effective polio vaccine was developed in 1952 by Jonas Salk at the University of Pittsburgh, but it would require years of testing. To encourage patience, Salk went on CBS radio to report a successful test on a small group of adults and children on March 26, 1953; two days later the results were published in JAMA ${ }^{20}$. The Salk vaccine, or inactivated poliovirus vaccine (IPV), is based on three wild, virulent reference strains, Mahoney (type 1 poliovirus), MEF-1 (type 2 poliovirus), and Saukett (type 3 poliovirus), grown in a type of monkey kidney tissue culture (Vero cell line), which are then inactivated with formalin. The injected Salk vaccine confers IgG-mediated immunity in the bloodstream, which prevents polio infection from progressing to viremia and protects the motor neurons, thus eliminating the risk of bulbar polio and post-polio syndrome.

Beginning February 23, 1954, the vaccine was tested at Arsenal Elementary School and the Watson Home for Children in Pittsburgh, Pennsylvania. Salk's vaccine was then used in a test called the Francis Field Trial, led by Thomas Francis; the largest medical experiment in history. The test began with some 4,000 children at Franklin Sherman Elementary School in McLean, Virginia, and would eventually involve 1.8 million children, in 44 states from Maine to California ${ }^{21}$. By the conclusion of the study, roughly 440,000 received one or more injections of the vaccine, about 210,000 children received a placebo, consisting of harmless culture media, and 1.2 million children received no vaccination and served as a control group, who would then be observed to see if any contracted polio.

The results of the field trial were announced April 12, 1955 (the tenth anniversary of the death of Franklin D. Roosevelt; see Franklin D. Roosevelt's paralytic illness). The Salk vaccine had been $60-70 \%$ effective against PV1 (poliovirus type 1), over $90 \%$ effective against PV2 and PV3, and 94\% effective against the development of bulbar polio. Soon after Salk's vaccine was licensed in 1955 children's vaccination campaigns were launched. In the U.S, following a mass immunization campaign promoted by the March of Dimes, the annual number of polio cases fell from 35,000 in 1953 to 5,600 by 1957 . By 1961 only 161 cases were recorded in the United States $^{22}$.

An enhanced-potency IPV was licensed in the United States in November 1987, and is currently the vaccine of choice in the United States. The first dose of polio vaccine is given shortly after birth, usually between 1-2 months of age; a second dose is given at 4 months of age. The timing of the third dose depends on the vaccine formulation but should be given between 6-18 months of age. A booster vaccination is given at 4 to 6 years of age, for a total of four doses at or before school entry. In some countries, a fifth vaccination is given during adolescence. Routine vaccination of adults (18 years of age and older) in developed countries is neither necessary nor recommended because most adults are already immune and have a very small risk of exposure to wild poliovirus in their home countries 23

In 2002, a pentavalent (5-component) combination vaccine (called Pediarix) containing IPV was approved for use in the United States. The vaccine also contains combined diphtheria, tetanus, and cellular pertussis vaccines $(\mathrm{DTaP})$ and a pediatric dose of hepatitis $B$ vaccine. In the UK, IPV is combined with tetanus, diphtheria, pertussis and Haemophilusinfluenzae type b vaccines. 
When the current formulation of IPV is used, $90 \%$ or more of individuals develop protective antibody to all three serotypes of poliovirus after two doses of inactivated polio vaccine (IPV), and at least $99 \%$ are immune to poliovirus following three doses. The duration of immunity induced by IPV is not known with certainty, although a complete series is thought to provide protection for many years ${ }^{24}$.

Oral vaccine: Oral polio vaccine (OPV) is a liveattenuated vaccine, produced by the passage of the virus through non-human cells at a sub-physiological temperature, which produces spontaneous mutations in the viral genome ${ }^{25}$. Oral polio vaccines were developed by several groups, one of which was led by Albert Sabin. Other groups, led by Hilary Koprowski and H.R. Cox, developed their own attenuated vaccine strains. In 1958, the National Institutes of Health created a special committee on live polio vaccines.

The various vaccines were carefully evaluated for their ability to induce immunity to polio, while retaining a low incidence of neuropathogenicity in monkeys. Large-scale clinical trials performed in the Soviet Union in late 1950s - early 1960 s by Mikhail Chumakov and his colleagues demonstrated safety and high efficacy of the vaccine.

Based on these results, the Sabin strains were chosen for worldwide distribution ${ }^{26}$. There are 57 nucleotide substitutions which distinguish the attenuated Sabin 1 strain from its virulent parent (the Mahoney serotype), two nucleotide substitutions attenuate the Sabin 2 strain, and 10 substitutions are involved in attenuating the Sabin 3 strain.

The primary attenuating factor common to all three Sabin vaccines is a mutation located in the virus's internal ribosome entry site (IRES) which alters stem-loop structures, and reduces the ability of poliovirus to translate its RNA template within the host cell. The attenuated poliovirus in the Sabin vaccine replicates very efficiently in the gut, the primary site of infection and replication, but is unable to replicate efficiently within nervous system tissue.

OPV also proved to be superior in administration, eliminating the need for sterile syringes and making the vaccine more suitable for mass vaccination campaigns. OPV also provided longer lasting immunity than the Salk vaccine ${ }^{27}$.
In 1961, type 1 and 2 monovalent oral poliovirus vaccine (MOPV) was licensed, and in 1962, type 3 MOPV was licensed. In 1963, trivalent OPV (TOPV) was licensed, and became the vaccine of choice in the United States and most other countries of the world, largely replacing the inactivated polio vaccine. A second wave of mass immunizations led to a further dramatic decline in the number of polio cases. Between 1962 and 1965 about 100 million Americans (roughly $56 \%$ of the population at that time) received the Sabin vaccine. The result was a substantial reduction in the number of poliomyelitis cases, even from the much reduced levels following the introduction of the Salk vaccine ${ }^{28}$.

OPV is usually provided in vials containing 10-20 doses of vaccine. A single dose of oral polio vaccine (usually two drops) contains 1,000,000 infectious units of Sabin 1 (effective against PV1), 100,000 infectious units of the Sabin 2 strain, and 600,000 infectious units of Sabin 3. The vaccine contains small traces of antibiotics- neomycin and streptomycin-but does not contain preservatives. One dose of OPV produces immunity to all three poliovirus serotypes in approximately $50 \%$ of recipients. Three doses of live-attenuated OPV produce protective antibody to all three poliovirus types in more than $95 \%$ of recipients. OPV produces excellent immunity in the intestine, the primary site of wild poliovirus entry, which helps prevent infection with wild virus in areas where the virus is endemic.

The live virus used in the vaccine is shed in the stool and can be spread to others within a community, resulting in protection against poliomyelitis even in individuals who have not been directly vaccinated. IPV produces less gastrointestinal immunity than does OPV, and primarily acts by preventing the virus from entering the nervous system. In regions without wild poliovirus, inactivated polio vaccine is the vaccine of choice.

In regions with higher incidence of polio, and thus a different relative risk between efficacy and reversion of the vaccine to a virulent form, live vaccine is still used. The live virus also has stringent requirements for transport and storage, which are a problem in some hot or remote areas. As with other live-virus vaccines, immunity initiated by OPV is probably lifelong $^{29}$. 
Latrogenic (Vaccine induced) polio: A major concern about the oral polio vaccine (OPV) is its known ability to revert to a form that can achieve neurological infection and cause paralysis. Clinical disease, including paralysis, caused by vaccinederived poliovirus (VDPV) is indistinguishable from that caused by wild polio viruses. This is believed to be a rare event, but outbreaks of vaccine-associated paralytic poliomyelitis (VAPP) have been reported, and tend to occur in areas of low coverage by OPV, presumably because the OPV is itself protective against the related outbreak strain ${ }^{30}$.

As the incidence of wild polio diminishes, nations transition from use of the oral vaccine back to the injected vaccine because the direct risk of iatrogenic polio (VAPP) due to OPV outweighs the indirect benefit of immunization via subclinical transmission of OPV. When IPV is used, reversion is not possible but there remains a small risk of clinical infection upon exposure to reverted OPV or wild polio virus. Following the widespread use of polio vaccines in the mid-1950s, the incidence of poliomyelitis declined rapidly in many industrialized countries. The use of OPV was discontinued in the United States in 2000 and in 2004 in the UK, but it continues to be used around the globe.

The rate of vaccine-associated paralytic poliomyelitis (VAPP) varies by region but is generally about 1 case per 750,000 vaccine recipients ${ }^{31}$.

VAPP is more likely to occur in adults than in children. In immunodeficient children, the risk of VAPP is almost 7,000 times higher, particularly for persons withB-lymphocyte disorders (e.g., agammaglobulinemia and hypogammaglobulinemia), which reduce the synthesis of protective antibodies. The World Health Organization considers the benefits of vaccination to far outweigh the risk of vaccine derived polio. Outbreaks of vaccine derived polio have been stopped by multiple rounds of high-quality vaccination, in order to immunize the entire population ${ }^{32}$. Outbreaks of VAPP occurred independently in Belarus (1965-66), Canada (196668), Egypt (1983-1993), Hispaniola (2000 2001), Philippines (2001),Madagascar (2001-2002), ( and in Haiti (2002), where political strife and poverty have interfered with vaccination efforts ${ }^{33}$. In 2006 an outbreak of vaccine-derived poliovirus occurred in China.
Cases have been reported from Cambodia (20052006), Myanmar (2006-2007), Iran (1995, 20052007), Syria, Kuwait and Egypt. Since 2005, The World Health Organization has been tracking vaccine-caused polio in northern Nigeria caused by a mutation in live oral polio vaccines ${ }^{34}$.

Epidemiology: As a result of vaccination, there has been a dramatic reduction in the incidence of poliomyelitis globally. However, the disease still remains endemic in four countries - Nigeria, India, Pakistan and Afghanistan. Other countries also have cases of wild-type poliomyelitis from time to time, due to importation. These efforts have reduced the number of annual diagnosed cases by $99 \%$; from an estimated 350,000 cases in 1988 to a low of 483 cases in 2001, after which it has remained at a level of about 1,000 cases per year $\left(1,606\right.$ in 2009) ${ }^{35}$.

The World Health Organization (WHO) aimed to eradicate poliomyelitis by the year 2005. Although this was not successful, WHO is still hopeful that polio eradication will be achieved by 2010 or soon after, primarily through 'national polio days' where all children in a certain region are given oral polio vaccine (OPV) Poliomyelitis has been a notifiable disease in Australia since 1922. The highest recorded incidence of poliomyelitis in the country (39.1 per 100,000 populations) was in 1938 . The last polio epidemic in Australia was in 1961-1962 ${ }^{36}$. Mass vaccination against polio using intramuscular Salk inactivated polio vaccine (IPV-Salk) first started in Australia in 1956. In 1966, IPV-Salk was replaced by Sabin oral polio vaccine (OPV-Sabin) in the publicly funded immunisation program. OPV is particularly suited to provide mass protection against wild-type polio. As expected, good vaccine coverage with OPV over several years led to cessation of indigenous transmission of wild polio virus infections in Australia $^{37}$.

The last reported case of locally acquired wild-type polio in Australia was in 1972. In 1986, there was a case of poliomyelitis reported in a 22 year old that was initially thought to be wild-type virus, but was later confirmed as a vaccine-like strain.8-10 In October 2000, Australia together with the other 36 countries in the Western Pacific Region was declared 'polio free' by the WHO. This certification of 'poliofree' status is confirmation of interruption of indigenous poliovirus transmission and containment of wild polio virus in the country ${ }^{38}$. 
Until global eradication of polio is achieved, all countries are at risk of polio infection. In 2005, Indonesia had an imported case of polio, 10 years after the last case of indigenously acquired poliomyelitis was reported in the country. This imported case of polio caused a re-establishment of local transmission of the virus and resulted in a large polio epidemic, with more than 200 cases $^{39}$.

As of 2012, polio remains endemic in only three countries: Nigeria, Pakistan, and Afghanistan, although it continues to cause epidemics in other nearby countries due to hidden or re-established transmission. For example, despite eradication ten years prior, an outbreak was confirmed in China in September of 2011 involving a strain prevalent in neighbouring Pakistan. Since January 2011, there were no reported cases of the disease in India, and hence in February 2012, the country was taken off the WHO list of polio endemic countries. It is reported that if there are no cases of polio in the country for two more years, it will be declared as a polio-free country ${ }^{40}$.

\section{LABORATORY DIAGNOSIS:}

1. Viral isolation: Poliovirus may be recovered from the stool or pharynx from a person with presumed poliomyelitis. Isolation of virus from the cerebrospinal fluid (CSF) is diagnostic but is rarely accomplished. If poliovirus is isolated from a person with acute flaccid paralysis, it must be tested further, using oligonucleotide mapping (fingerprinting) or genomic sequencing, to determine if the virus is wild-like or vaccine-like.

2. Serology: Neutralizing antibodies appear early and may be at high levels by the time the patient is hospitalized and, therefore, a 4-fold rise may not be demonstrated.

3. Cerebrospinal fluid: The CSF in poliovirus infection usually contains an increased number of white blood cells (10 to 200cells/mm3, primarily lymphocytes) and a mildly elevated protein from 40 to $50 \mathrm{mg} / 100 \mathrm{ml}$.

\section{PREVENTION}

1. Passive immunization: In 1950, William Hammon at the University of Pittsburgh purified the gamma globulin component of the blood plasma of polio survivors. Hammon proposed the gamma globulin, which contained antibodies to poliovirus, could be used to halt poliovirus infection, prevent disease, and reduce the severity of disease in other patients who had contracted polio. The results of a large clinical trialwere promising; the gamma globulin was shown to be about $80 \%$ effective in preventing the development of paralytic poliomyelitis. It was also shown to reduce the severity of the disease in patients who developed polio. The gamma globulin approach was later deemed impractical for widespread use, however, due in large part to the limited supply of blood plasma, so the medical community turned its focus to the development of a polio vaccine ${ }^{41}$.

2. Vaccine: Two types of vaccine are used throughout the world to combat polio. Both types induce immunity to polio, efficiently blocking person-to-person transmission of wild poliovirus, thereby protecting both individual vaccine recipients and the wider community (socalled herd immunity) ${ }^{42}$. The first candidate polio vaccine, based on one serotype of a live but attenuated (weakened) virus, was developed by the virologist Hilary Koprowski. Koprowski's prototype vaccine was given to an eight-year-old boy on February 27, 1950. Koprowski continued to work on the vaccine throughout the 1950s, leading to large-scale trials in the then Belgian Congo and the vaccination of seven million children in Poland against serotypes PV1 and PV3 between 1958 and $1960^{43}$.

The second inactivated virus vaccine was developed in 1952 by Jonas Salk at the University of Pittsburgh, and announced to the world on April 12, 1955. The Salk vaccine, or inactivated poliovirus vaccine (IPV), is based on poliovirus grown in a type of monkey kidney tissue culture (vero cell line), which is chemically inactivated with formalin. After two doses of IPV (given by injection), $90 \%$ or more of individuals develop protective antibody to all three serotypes of poliovirus, and at least $99 \%$ are immune to poliovirus following three doses 44.

Subsequently, Albert Sabin developed another live, oral polio vaccine (OPV). It was produced by the repeated passage of the virus through 
nonhuman cells at sub physiological temperatures. The attenuated poliovirus in the Sabin vaccine replicates very efficiently in the gut, the primary site of wild poliovirus infection and replication, but the vaccine strain is unable to replicate efficiently within nervous system tissue. A single dose of Sabin's oral polio vaccine produces immunity to all three poliovirus serotypes in about $50 \%$ of recipients.

Three doses of live-attenuated OPV produce protective antibody to all three poliovirus types in more than $95 \%$ of recipients. Human trials of Sabin's vaccine began in 1957, and in 1958 it was selected, in competition with the live vaccines of Koprowski and other researchers, by the US National Institutes of Health. Licensed in 1962 , it rapidly became the only polio vaccine used worldwide ${ }^{45}$.

\section{NATIONAL IMMUNISATION PROGRAM (NIP):}

Children: Children are recommended a primary course of 3 doses of an IPV-containing vaccine at 2, 4 and 6 months of age and a booster dose at 4 years of age. The recommended interval between 2 doses is 2 months, but, for catch-up, the minimum interval can be 1 month. In the Australian setting, a 3-dose primary schedule and a booster at 4 years of age provides adequate protection. Therefore, for those children who have received a complete course of polio vaccine during childhood, a further booster dose is not required later in life unless they are at increased risk of infection as below.

Adults: The schedule for unvaccinated adults is 3 doses administered at intervals of 1-2 months.

Booster doses: A booster dose is not required for fully vaccinated children or adults unless they are at increased risk of infection, such as travelling to areas or countries where poliomyelitis is epidemic or endemic healthcare workers, including laboratory workers, in possible contact with poliomyelitis cases. For those exposed to a continuing risk of infection, booster doses are desirable every 10 years. There is no cure for polio. The focus of modern treatment has been on providing relief of symptoms, speeding recovery and preventing complications. Supportive measures include antibiotics to prevent infections in weakened muscles, analgesics for pain, moderate exercise and a nutritious diet.
Treatment of polio often requires long-term rehabilitation, including physical therapy, braces, corrective shoes and, in some cases, orthopedic surgery ${ }^{46}$.

Portable ventilators may be required to support breathing. Historically, a non-invasive, negativepressure ventilator, more commonly called an iron lung, was used to artificially maintain respiration during an acute polio infection until a person could breathe independently (generally about one to two weeks). Today, many polio survivors with permanent respiratory paralysis use modern jacket-type negative-pressure ventilators worn over the chest and abdomen $^{47}$.

Other historical treatments for polio include hydrotherapy, electrotherapy, massage and passive motion exercises, and surgical treatments, such as tendon lengthening and nerve grafting. Devices such as rigid braces and body casts-which tended to cause muscle atrophy due to the limited movement of the user-were also touted as effective treatments ${ }^{48}$.

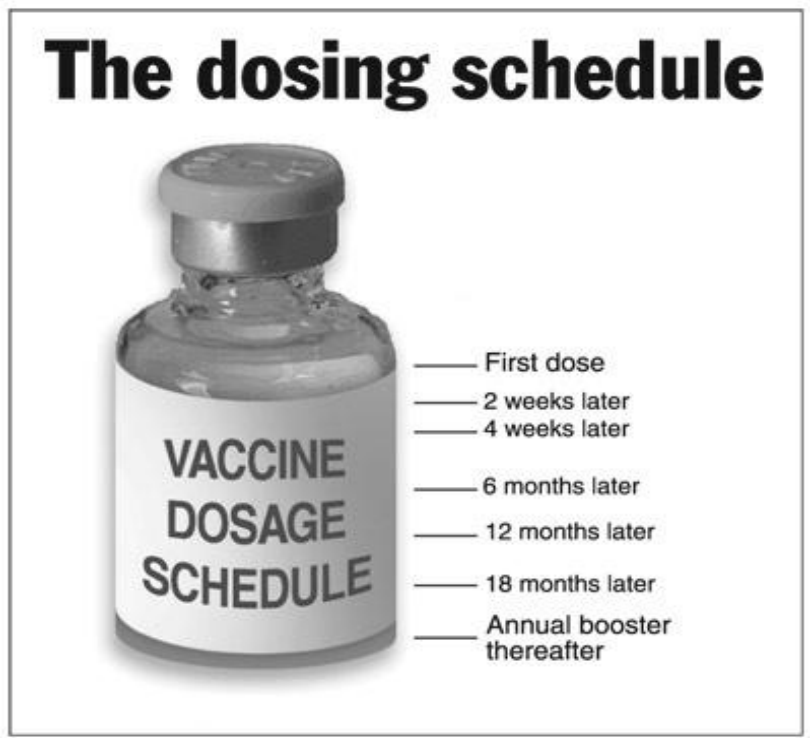

FIG. 2: THE DOSING SCHEDULE OF POLIO VACCINE

Natural Ayurvedic Treatment of Polio: Polio is a very deadly virus and though partly eradicated through an extensive worldwide immunization programme it is still around and wreaking its deadly havoc upon parts of the population. It is a highly infectious disease and enters the body through the mouth and goes onto attack the central nervous system. The cause of this despite extensive medical research has yet to be found. Though many who catch the virus survive most that do are left with a form of paralysis, usually in an arm or a leg. 
Though it is unable to offer a cure, Ayurvedic can alleviate the pain experienced by sufferers and can also aid survivors' recovery greatly.

Ayurvedic Cause of Polio: Paralysis or more specifically polio is caused according to Ayurvedic when there is a divergence in vata-pitta-kamba which affects the dhosa (energies of the body). This physiological shift causes toxins (ama) to build up and attack the nervous system. To be treated positively Ayurvedic says that there must be a rebalancing of these within the body. To achieve this state the following methods should be applied.

Ayurvedic Remedies for Polio: As previously stated any form of the Ayurvedic programme can not cure polio. But what it can do is offer a healing regime that repairs the damage wrought by the disease. This takes two forms Herbal remedy and intensive Ayurvedic massaging. The herbal remedies can eradicate internal disorder and strengthen the immune system.

Massage alleviates the on-going pain and any paralysis that may have occurred. In this instance intensive massages repair the dead and wasted muscle. Massaging also allows the affected area or joint to receive nutrients which can expel toxins.

\section{Massage applied to remedy polio in Ayurvedic:} As possibly befits the remedy for such a deadly serious disease like polio, Ayurvedic prescribes arguably its' most important massage form in the treatment of the disease, namely the method known as Pindasvedka or Navarakizhi. This treatment revitalizes mainly the skin and is also used with Ayurvedic anti-ageing treatments. However in achieving this it also rejuvenates dead and wasted muscles which are why it is used as a remedy for paralysis, which is why it used on polio sufferers.

\section{The revitalizing agent in Pindasvedka or} Navarakizhi: Pindasvedka or Navarakizhi is a simple remedy consisting of medicated rice. The rice provides carbohydrates which rejuvenate the affected areas when applied. Pindasvedka or Navarakizhi is applied in Ayurvedic

A fine cloth of the medicated rice solution is placed upon the patients' body. This creates gradual warmth and is applied until the patient builds up a great sweat. The act of sweating expels the toxins and in doing so begins to rejuvenate the affected areas.
The Ayurvedic way to alleviate the pain caused by polio and to treat it is mainly massage based. But the imbalances caused by the pitta-vatta-kamba shift that disrupts the internal systems of a polio sufferer can be put right by ingesting certain herbs and spices. They can be added to cooking and will aid the healing processes of paralysis sufferers.

Herbs and spices recommended in the Ayurvedic cure for polio

1. Fennel

2. Ginger

3. Black pepper

4. Coriander

5. Licorice

CONCLUSION: The above review revels that rehabilitation in patients with post-polio syndrome should take a multi professional and multidisciplinary approach, with an emphasis on physiotherapy, including enhanced or individually modified physical activity, and muscle training. Patients with post-polio syndrome should be advised to avoid both inactivity and over use of weak muscles. Evaluation of the need for orthoses and assistive devices is often required.

\section{REFERENCES:}

1. Cohen JI: "Chapter 175: Enteroviruses and Reoviruses". Harrison's Principles of Internal Medicine. McGraw-Hill Professional. Edition 16, 2004: 1144-1145. ISBN 0-07140235-7.

2. Atkinson W, Hamborsky J, McIntyre L and Wolfe S: "Poliomyelitis" (PDF).Epidemiology and Prevention of Vaccine-Preventable Diseases (The Pink Book). Washington DC: Public Health Foundation. Edition 11, 2009: 231-44.

3. Trevelyan B, Smallman-Raynor M and Cliff A: The Spatial Dynamics of Poliomyelitis in the United States: From Epidemic Emergence to Vaccine-Induced Retreat. 2005; 2: 269-93. PMC 1473032.PMID 16741562.

4. Heymann D: Global polio eradication initiative .Bull. World Health Organ. 2006; 84 (8): 595. PMC 2627439.PMID 16917643.

5. Ohri, Linda K, Jonathan G and Marquess: Polio: Will We Soon Vanquish an Old Enemy? Drug Benefit Trends. 1999; 11 (6): 41-54.

6. Kew O, Sutter R, de Gourville E, Dowdle W and Pallansch $\mathrm{M}$ : "Vaccine-derived polioviruses and the endgame strategy for global polio eradication". Annu Rev Microbiol. 2005; 59: 587-635.

7. Mueller S, Wimmer E and Cello J: "Poliovirus and poliomyelitis: a tale of guts, brains, and an accidental event". Virus Res. 2005; 111 (2): 175-93.

8. Silverstein A, Silverstein V and Nunn LS: Polio. Diseases and People. Berkeley Heights, NJ: Enslow Publishers. 2001:12. ISBN 0-7660-1592-0. 
9. Gawne AC and Halstead LS: Post-polio syndrome: pathophysiology and clinical management. Critical Review in Physical Medicine and Rehabilitation. 1995; 7: 147-88.

10. Nathanson $\mathrm{N}$ and Martin $\mathrm{J}$ : The epidemiology of poliomyelitis: enigmas surrounding its appearance, epidemicity, and disappearance. Am J Epidemiol. 1979; 110 (6): 672-92.

11. Cono J and Alexander LN: Chapter 10, Poliomyelitis. Vaccine Preventable Disease Surveillance Manual. Centers for Disease Control and Prevention. Edition 3, 2002:10-1.

12. Yin-Murphy $\mathbf{M}$ and Almond JW: Picornaviruses: The Enteroviruses: Polioviruses. Baron's Medical Microbiology. Univ. of Texas Medical Branch. Edition 4, 1996: 8791. ISBN 0-9631172-1-1.

13. Professional Guide to Diseases (Professional Guide Series). Hagerstown, MD: Lippincott Williams \& Wilkins. 2005: 243-5. ISBN 1-58255-370-X.

14. Hoyt, William G, Miller N and Walsh F: Walsh and Hoyt's clinical neuro-ophthalmology. Hagerstown, MD: Lippincott Williams \& Wilkins.2005: 3264-65. ISBN 0-7817-4814-3.

15. Yin-Murphy $M$ and Almond JW: Picornaviruses: The Enteroviruses: Polioviruses. Baron's Medical Microbiology. Univ. of Texas Medical Branch. Edition 4, 1996: 7578. ISBN 0-9631172-1-1.

16. Mueller S, Wimmer E and Cello J: Poliovirus and poliomyelitis: a tale of guts, brains, and an accidental event. Virus Res. 2005; 111 (2): 175-93.

17. A Science Odyssey: People and Discoveries. PBS. 1998. Retrieved 2008-11-29.

18. Kew O, Sutter R, de Gourville E, Dowdle W and Pallansch $\mathrm{M}$ : Vaccine-derived polioviruses and the endgame strategy for global polio eradication. AnnuRev. Microbiol. 2005; 59:587-635.

19. Agarwal SP: Pharmaceutical Jurisprudence \& Ethics, Birla publications. 1999:142.

20. Offit and Paul A : The Cutter Incident: How America's First Polio Vaccine Led to the Growing Vaccine Crisis. Yale University Press. 2007:38. ISBN 0-300-12605-0

21. Polio Victory Remembered as March of Dimes Marks 50th Anniversary of Salk Vaccine Field Trials. News Desk. 200404-26. Archived from the original on 2008-09-19. Retrieved 2008-11-29.

22. Hinman A: Landmark perspective: Mass vaccination against polio. JAMA. 1984; 251 (22): 2994-6.

23. Atkinson W, Hamborsky J, McIntyre L and Wolfe S: Epidemiology and Prevention of Vaccine-Preventable Diseases (The Pink Book). Washington, D.C.: Public Health Foundation. Edition 10, 2008: 27-31.

24. Robertson, Susan. "Module 6: Poliomyelitis" (PDF). The Immunological Basis for Immunization Series. World Health Organization (Geneva, Switzerland). Retrieved 2008-11-29.

25. Sabin AB: Role of my cooperation with Soviet scientists in the elimination of polio: possible lessons for relations between the U.S.A. and the USSR. Perspect. Biol. Med. 1987; 31 (1): 57-64. PMID 3696960

26. Ochs K, Zeller A and Saleh L: Impaired Binding of Standard Initiation Factors Mediates Poliovirus Translation Attenuation. J. Virol. 2003; 77 (1): 115-22.
27. Smallman-Raynor and Matthew: Poliomyelitis: A World Geography: Emergence to Eradication. Oxford University Press, USA. 2006: 453. ISBN 0-19-924474-X.

28. Poliomyelitis Eradication: Field Guide. Washington: Pan American Health Organization. 2006. ISBN 92-75-11607-5

29. Shimizu H, Thorley B and Paladin FJ: Circulation of Type 1 Vaccine-Derived Poliovirus in the Philippines in 2001. J. Virol. 2004; 78 (24): 13512-21.

30. Racaniello V: One hundred years of poliovirus pathogenesis. Virology. 2006; 344 (1): 916.

31. "What is vaccine-derived polio?". WHO. 2007-10-08. Retrieved 2008-11-29

32. Kew O, Wright $\mathrm{P}$ and Agol V: Circulating vaccine-derived polioviruses: current state of knowledge. Bull World Health Organ. 2004; 82 (1): 16-23.

33. Liang $\mathrm{X}$, Zhang $\mathrm{Y}$ and $\mathrm{Xu} \mathrm{W}$ : An outbreak of poliomyelitis caused by type 1 vaccine-derived poliovirus in China. $\mathrm{J}$ Infect Dis. 2006; 194 (5): 545-51.

34. Centers for Disease Control and Prevention (CDC): Update on vaccine-derived polioviruses. MMWR Morb Mortal Wkly Rep. 2006; 55 (40): 1093-7. PMID 17035927

35. Centers for Disease Control and Prevention (CDC): "Progress toward interruption of wild poliovirus transmission-worldwide, January 2007-April 2008".MMWR Morb. Mortal. Wkly. Rep. 2008; 57 (18): 489-94.PMID 18463607.

36. Roche P and Spencer J: Polio eradication in Australia and the world [editorial]. Communicable Diseases Intelligence 2002; 26: 113-117.

37. D' Souza RM, Kennett M and Watson C: Australia declared polio free. Communicable Diseases Intelligence. 2002; 26:253-260.

38. Thorley BR, Bussan KA, Elliott EJ and Kelly HA: Vigilance is required for Australia to remain polio free. Medical Journal of Australia. 2006; 184:474-475.

39. Fine PEM: "Polio: Measuring the protection that matters most". J Infect Dis. 2009; 200 (5): 673-675.

40. Hammon W: Passive immunization against poliomyelitis. MonogrSer World Health Organ. 1995; 26: 357-70.PMID 14374581.

41. Fine $\mathrm{P}$ and Carneiro I: Transmissibility and persistence of oral polio vaccine viruses: implications for the global poliomyelitis eradication initiative. Am $\mathbf{J}$ Epidemiol. 1999; 150 (10): 1001-21. PMID 10568615

42. Koprowski and Hilary: Interview with Hilary Koprowski, sourced at History of Vaccines website. College of Physicians of Philadelphia. 2010: 34- 37.

43. Spice B: The Salk vaccine: 50 years later/ second of two parts (Pittsburgh Post-Gazette). 2005: 567-8.

44. Sabin $\mathrm{AB}$ and Boulger LR: History of Sabin attenuated poliovirus oral live vaccine strains. J Biol Stand. 1973; 1 (2): 115-8.

45. Daniel, TM and Robbins FC: Polio. Rochester, N.Y., USA: University of Rochester Press. 1997: 8-10. ISBN 1-58046066-6.

46. Goldberg A: Noninvasive mechanical ventilation at home: building upon the tradition". Chest. 2002; 121 (2): 321-24.

47. Oppewal S: Sister Elizabeth Kenny, an Australian nurse, and treatment of poliomyelitis victims. Image J Nurs Sch. 1997; 29 (1): 83-7.

How to cite this article:

Singh R, Monga AK and Bais S: Polio: A Review. Int J Pharm Sci Res 2013; 4(5); 1714-1724. 